\title{
Social and economic burden of patients with influenza-like illness and clinically diagnosed flu treated at various health facilities in Vietnam
}

This article was published in the following Dove Press journal:

ClinicoEconomics and Outcomes Research

19 July 2017

Number of times this article has been viewed

\author{
Trung Quang Vo ${ }^{1,2}$ \\ Usa Chaikledkaew ${ }^{1,3}$ \\ Minh Van Hoang ${ }^{4}$ \\ Arthorn Riewpaiboon' \\ 'Department of Pharmacy, Division \\ of Social and Administrative \\ Pharmacy, Faculty of Pharmacy, \\ Mahidol University, Bangkok, \\ Thailand; ${ }^{2}$ Department of Pharmacy \\ Administration, Faculty of Pharmacy, \\ University of Medicine and Pharmacy, \\ Ho Chi Minh City, Vietnam; ${ }^{3}$ Health \\ Intervention and Technology \\ Assessment Program (HITAP), \\ Ministry of Public Health, Nonthaburi, \\ Thailand; ${ }^{4}$ Hanoi School of Public \\ Health, Hanoi, Vietnam
}

\begin{abstract}
Influenza is a malady related to the human respiratory system, and its influenza-like illness (ILI) can cause an economic and social burden, especially among children and the elderly. This study was conducted to estimate the cost of illness based on a social perspective of ILI cases. A prospective study was conducted between February and March of 2016 in pharmacies, private clinics at Khanh Hoa, Lam Dong, Tay Ninh, Dong Nai Province, and Ho Chi Minh City, and Hospital of Tropical Diseases. Demographic and clinical information were collected by face-to-face interview. Direct costs (e.g., diagnosis and therapeutics) and indirect costs (e.g., cost of day loss) are included as treatment costs. The average cost of treatment associated with ILI was US\$ 88.09 per case for all age groups; direct non-medical cost was higher compared to direct medical cost: $39.5 \%$ in pharmacies, $71.1 \%$ in clinics, and $64.2 \%$ in hospital. The indirect cost was US\$27.49 per episode. The average total cost for children below 14 years old was much higher than that for the other age groups. The cost of illness of ILI was, therefore, the reason for the economic burden of influenza patients and their families. This study provides a database for future research and programs, and policies that can be adopted for influenza or ILI in Vietnam.
\end{abstract} Keywords: influenza, influenza-like illness, economic burden, cost of illness, pharmacy, clinic, hospital, Vietnam

\section{Introduction}

Influenza, or flu, is a malady related to the human respiratory system and is known to be caused by the influenza virus. However, illnesses with influenza-like symptoms are likely to be the result of several types of viruses, which doctors find difficult to specifically match up with their corresponding sickness. Thus, such illnesses are labeled as influenza-like illness (ILI). Flu is known to be considerably infectious and mainly spreads through coughs and sneezes of an infected person. For many patients, complications and sequelae are uncommon results of influenza and ILI. The onset of symptoms caused by naturally acquired infection is generally fast with visible signs of fever, chills, myalgia, headache, malaise, sore throat, cough - which rarely exceed a time limit of 1 week $^{1}$ - muscle and joint pain and running nose. Influenza epidemics are believed to be responsible for severe illness or mortality, especially in individuals of high-risk groups and vulnerable subjects, for example, children, pregnant women, and the elderly. Ceaseless evolution is a noticeable feature of influenza viruses. Hence, humans with average immunity can be infected multiple times throughout their life. ${ }^{2}$ Moreover, influenza virus spreads not only via respiratory tract but also via body contact between the healthy and the infected people (e.g., by shaking hands). Once
Correspondence: Arthorn Riewpaiboon Department of Pharmacy, Division of Social and Administrative Pharmacy, Faculty of Pharmacy, Mahidol University, 447, Sri Ayutthaya Road, Bangkok 10400, Thailand

Tel +66 2644867790 Ext 5745

Fax +6626448694

Email arthorn.rie@mahidol.ac.th 
infected with influenza, an individual will become immediately contagious, but will not be aware of the infection until they develop symptoms the following day or up to 7 days later. A flu epidemic on a national level with great number of infected cases could last for several weeks. ${ }^{3}$ The World Health Organization (WHO) has estimated the annual worldwide attack rate of influenza to be $\sim 10-15 \%$, which varies within the range of $5-10 \%$ for adults and $20-30 \%$ for children. Furthermore, the yearly epidemics are believed to cause severe illness in $\sim 3-5$ million cases and deaths in $\sim 250-500$ million cases per annum, worldwide. ${ }^{2}$ In the winter epidemics of recent years, influenza-attributable deaths in England and Wales (all causes, all age-groups) have been estimated to be at an average of 12,000 per annum. ${ }^{4}$ In the USA, 24.7 million cases of influenza per annum with an approximate number of 31.4 million outpatient visits were recorded based on 2003 population demographics. ${ }^{5}$

Influenza spreads rapidly around the world and creates a considerable economic and social burden. The health care-directed costs should be the first to be focused on. For example, the predicted cost of the Australian health care system for influenza/ILI was AU\$115 million per annum. ${ }^{6}$ In the USA, medical costs totaled US\$3,990 in 2012 for inpatients and US\$730 for emergency department patients. ${ }^{7}$ In Belgium, the average direct medical cost per case in 2014 was $€ 51-53 .{ }^{8}$ From a societal viewpoint, the total average mean cost for physician-diagnosed influenza and ILI at primary care level in Germany in 2015 was $€ 514$ for adults and $€ 105$ for children. ${ }^{9}$ ILI costs are not only direct medical cost but also indirect cost of work days lost for both patients and their family. So from a community perspective, society has paid for influenza treatment and its related cost. For instance, in Australia, of the mean ILI cost, AU\$406 was due to loss of work days, AU\$102 due to health care visits, and AU\$118 due to other factors such as medications and missed childcare attendance. The average hours of work missed due to ILI were 13 work hours and 3 leisure hours per ILI. ${ }^{10}$ Meanwhile in the US, the lost work hours that caregivers of hospitalized children experienced was estimated at an average time span of 73 hours, costing US\$1,456. Caregivers of children who were admitted into the emergency department and outpatient clinics were reported to miss a large number of work hours with 19 work hours (US\$383) and 11 work hours (US\$222), respectively. ${ }^{7}$

The disease burden of ILI in most tropical and subtropical countries has not been described adequately till date. Hence, the aim of this study was to explore the treatment cost of ILI and clinically diagnosed influenza from a social perspective.

\section{Materials and methods Case definitions}

In this study, self-reported ILI was identified as fever with a body temperature $>38^{\circ} \mathrm{C}$ and with symptoms of either cough or sore throat. Meanwhile, several of the subjects believed that their illness was a common fever with symptoms of cough and sore throat. Nevertheless, the said cases were not eliminated. ${ }^{11}$

\section{Study design}

This analysis focused on the cross-sectional cost of illness (COI); data obtained from surveys conducted in Vietnam were used to evaluate the cost per influenza or ILI episode. COI study measures the economic burden of a disease for a given period of time. From a social perspective, all costs, regardless of cases incurred, are included. Thus, costs to the health care service, to social services, to patients, and also to the rest of the society in the form of production losses are included.

\section{Study population and sampling method}

Vietnam has a tropical monsoon climate and an average temperature of $24.3^{\circ} \mathrm{C} .{ }^{12}$ However, the climate tends to vary considerably from place to place. The average annual temperature is generally higher in the plains than in the plateaus and higher in the southern parts than in the northern parts. According to the General Statistics Office of Vietnam (2014), the population of Vietnam is 90.7 million. ${ }^{13}$

A sample of 15 pharmacies, 3 clinics, and 1 hospital were involved in this study for the convenience of collecting samples. A cross-sectional study was conducted to select ILI impact data in March 2016 at Ho Chi Minh City and in the 4 provinces of Southern Vietnam: Khanh Hoa, Lam Dong, Tay Ninh, and Dong Nai (Figure 1).

\section{Calculation of sample size and sample assignment}

The sample size was calculated by calculating unidentified population. The minimum sample size can be calculated based on the following formula ${ }^{14}$ (simple random sampling):

$$
n=\frac{z^{2} * N * p^{*}(1-p)}{d^{2} * N+z^{2} * p^{*}(1-p)}
$$

Where $N=$ the total population of Vietnam, $p=$ the proportion of patients with ILI symptoms, $d=$ the rate of allowable 


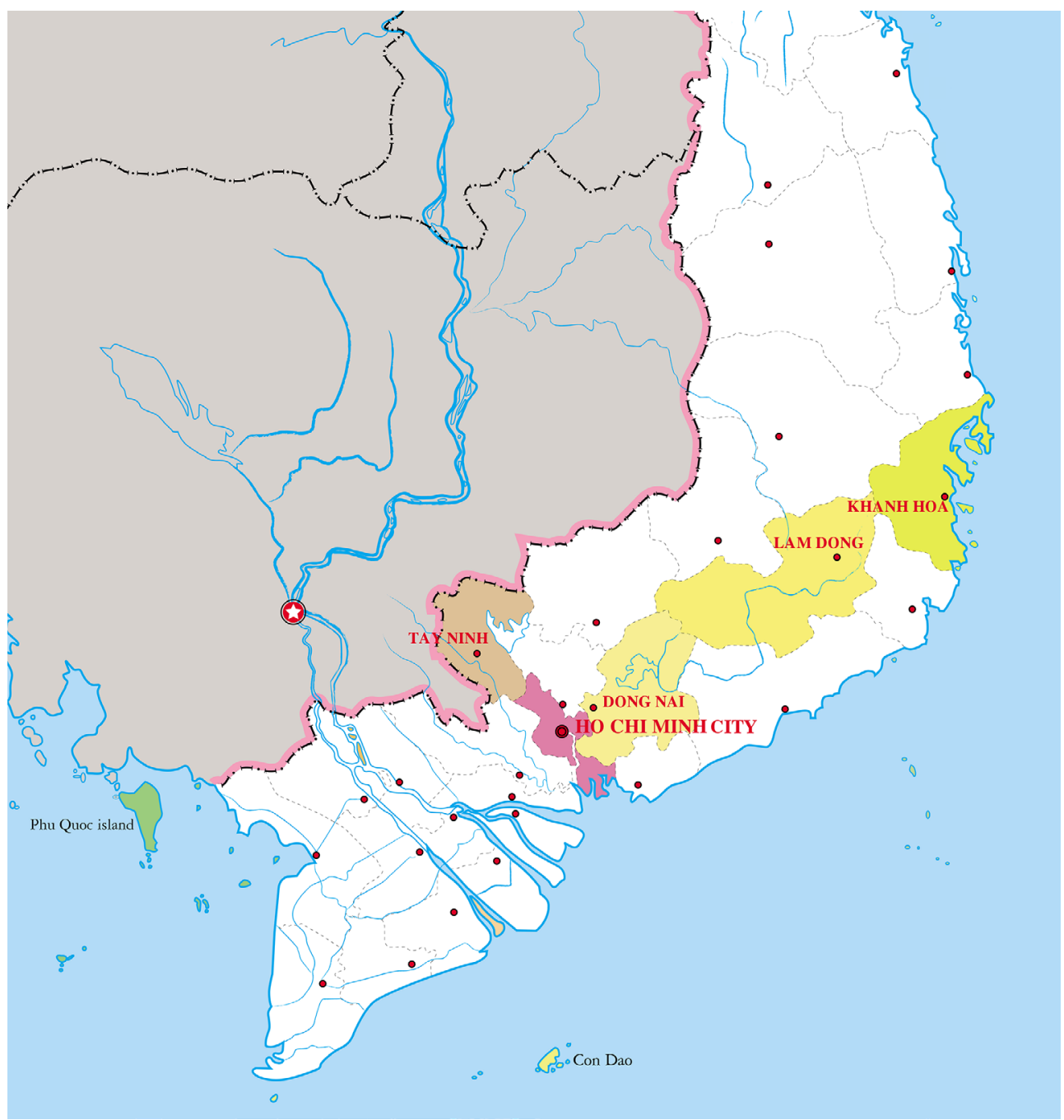

Figure I Map of provinces surveyed for data collection in Southern Vietnam.

error, and $z=$ the standard normal deviation at $95 \% \mathrm{CI}$ (confidence interval).

Using the aforementioned formula, the sample size obtained should be equal to:

$$
n=\frac{N}{N \cdot d^{2}+1}=\frac{469,353}{469,353 \cdot(0.05)^{2}+1}=399.66
$$

Where $N=90,700,000$ (total population of Vietnam in 2015), ${ }^{13} d=5 \%$ (allowable error). It should be reemphasized that the sample size here is assumed based on random sampling, and $p=0.5$ (used as a normal distribution of $50 \%$ ).

To eliminate errors in the sampling surveys, the sample size was increased by a factor of $110 \%$ to allow for lack of follow-up and non-response. All factors were used based on expert opinion. In total, it was anticipated that 440 participants would be surveyed in this study.

\section{Data collection}

Questionnaires were modified from World Health Organization Manual for Estimating the Economic Burden of Seasonal Influenza $^{15}$ (Supplementary materials). All patients or their caregivers were invited to participate in the survey, in which they received a covering letter and questionnaires. All participants were surveyed in health care facilities, where they received treatment. The study included three steps and are as follows:

\section{Step I: selection of participants and obtaining their approval}

The patient survey was administered by researchers, who underwent 1-day training on the methods for data collection. 
They were responsible for data collection at pharmacies, clinics, and outpatient department of a hospital. A group of 40 senior pharmacy students were selected and were allocated to health care facilities, with 2 students per facility, to interview the patients.

Each patient or members of their family were interviewed for their knowledge on the illness and the relevant costs of treatment of ILI symptoms. Afterward, the subjects were divided into two groups based on their symptoms: ILI patients or patients with ILI symptoms (one or more symptoms but not ILI) based on the diagnosis of a medical doctor. All subjects were enrolled from February to March in 2016 and completed a registration form requiring demographic information.

\section{Step 2: face-to-face survey}

The interviews were conducted by a trained surveyor with a focus on ILI patients. The collected information was then divided into three parts.

- Part 1: General information of participant (age, gender, area of residence, education, occupation, monthly income, health insurance status, self-medication, quality of life, history of influenza, the day first symptom appeared, and average members in the family).

- Part 2: COI including treatment before admission, treatment during admission, and treatment after discharge (direct medical cost, direct non-medical cost, and indirect cost).

- Part 3: COI for different age groups.

\section{Step 3: follow-up telephone interview}

An ILI patient or a caregiver was interviewed via telephone 7 days after the onset of illness for information about influenza symptoms to estimate effective treatment, time required for a patient to completely recover from illness, number of health care visits, cost of treatment, meals (food that a patient and caregiver ate during the treatment period), transportation cost (Supplementary materials).

\section{Characteristic of participants}

This patient survey investigated the incidence of ILI on a major scale of population with many constituent factors (different genders, age, education levels, occupations, and income) living in urban or rural areas in Vietnam. In addition patients were selected in different health care centers such as pharmacies, private clinics, and hospital, which were located in either city or other provinces.

\section{Medical care costs of ILI}

Costs were divided into two categories: direct costs and indirect costs. Direct costs were then divided into two subcategories: direct medical cost and direct non-medical cost, in which the former included expenses such as on diagnosis (e.g., radiology and laboratory), therapeutics (e.g., pharmaceuticals, blood products, and intensive care therapies), prescription, drugs, hospital services.

Additionally, the latter included cost of transportation, meal, accommodation, and caregiver, who were also questioned about the uses and sources of time lost to take care patients such as the number of days spent on doctor visits, hospitalization stay or home care during the child's illness was used to account for the uses of caregivers' time. For caregivers, efforts were made to identify whether the time lost was from work, school or college, or other activities.

Meanwhile, indirect cost was calculated by the cost of lost days, about which ILI patients were to answer during the interview. Depending on the individuals, the definition of the "lost day" differed, for example, personal leave at work for employed individuals and absent school days for students or preschoolers (kindergarten/childcare center goers). The indirect cost of an individual is calculated as the daily income/ expense multiplied by the lost days due to illness.

\section{Methods of cost calculation (direct and indirect costs)}

Specifically, on national-level scale, the summation of costs of every influenza case is likely to result in economic impacts. Total economic impacts were calculated by total direct cost plus total indirect cost. To be more specific, such impacts were the results of the summation between direct medical cost, direct non-medical cost, and indirect cost.

\section{Statistical analysis}

Several aspects of resources, utilization rates, and costs for each case were calculated. Estimates within each outcome differed by age group in pharmacies, clinics, and hospital. Microsoft Excel 2010 was used to calculate the data and Adobe Photoshop software to edit Figure 1.

\section{Ethics statement}

Written informed consent was obtained from adult participants and from parents or guardians on behalf of children participants during the study and before specimen collection and questionnaire survey. 
This study was carried out with the approval of the Biomedical Research Ethics Council at the University of Medicine and Pharmacy, Ho Chi Minh City, Vietnam.

\section{Results}

\section{Overview of patient demographics who were surveyed in various health facilities in Vietnam}

In total, 658 participants with ILI were surveyed in 2016; information regarding their age, gender, area of residence (rural/urban), education, occupation, income per month, self-medication, vaccination, history of influenza, and average number of family members of the surveyed subjects are shown in Table 1.

Among the total patients surveyed, 456 were surveyed at pharmacies, among whom most were adults (25-64 years) $(\mathrm{n}=283 ; 61.8 \%)$. Meanwhile, those surveyed at clinics and the hospital were mainly children $(92.1 \%$ in clinics and $74.5 \%$ in the hospital). The number of senior patients ( $>64$ years) accounted for negligible proportions with only 16 cases $(3.5 \%)$ in pharmacies, 1 case $(2 \%)$ in hospital, and no patient in private clinics.

As shown in Table 1, the ratio of men and women interviewed was even. Specifically, the number of females interviewed was $269(59.0 \%)$ in pharmacies, $91(60.3 \%)$ in clinics, and 23 (45.1\%) in hospital. Regarding the area of residence, most patients lived in rural, especially those who were surveyed at pharmacies $(n=284 ; 62.3 \%)$ and clinics $(n=100$; 66.2\%). Apart from these, most patients surveyed in hospital lived mainly in cities $(\mathrm{n}=35 ; 68.6 \%)$. In addition, the average number of family members who took care of the patients in three groups was 4.2. Table 1 also shows that the mean income of the interviewed patients was US\$208.2, with which most of the patients felt economically safe. For instance, at pharmacies, 231 out of 275 patients had incomes below US $\$ 250$ per month and were satisfied with their incomes; similarly, the number of patients satisfied with their income was 130 out of 135 in clinics and 34 out of 37 in hospital. Meanwhile, patients with high incomes (>US\$700 per month) also felt satisfied: 10 out of 12 patients at pharmacies and $100 \%$ of patients in clinics with incomes above US\$700 were satisfied.

Among the 658 patients, students and kindergarten children were found to be in higher numbers than the others, with their population being 178 and 147 people, respectively. Among the patients surveyed at pharmacies, students have the highest proportion $(\mathrm{n}=105 ; 3.0 \%)$ followed by laborer/ employee $(n=96 ; 21.1 \%)$, or private employee $(n=96 ; 21.1 \%)$. On the contrary, in clinics and hospital, the majority of patients were kindergarten children and accounted for 55.6\% $(n=84)$ and $62.7 \%(n=32)$, respectively, compared to the total population. A significant difference in the number of patients belonging to different education levels was noted and reported. Most of the patients were illiterate $(n=158)$, followed by high school students $(\mathrm{n}=137)$ and students with a bachelor degree and above $(\mathrm{n}=111)$. High school students interviewed at pharmacies $(\mathrm{n}=126 ; 27.6 \%)$ were the largest number of patients interviewed, followed by illiterate patients in clinics $(\mathrm{n}=102 ; 67.5 \%)$ and hospital $(\mathrm{n}=27 ; 52.9 \%)$. The first symptoms appeared in an average of 3 days. Out of the total 456 patients interviewed at pharmacies, 171 (37.5\%) had not had influenza in their lifetime until now. Otherwise, the numbers of patients who did not have a medical history regarding ILI were $82(54.3 \%)$ in clinics and $30(58.8 \%)$ in hospital. In summary, nearly half of the 658 patients who were interviewed indicated that they had previously had influenza. The mean duration of latest ILI noted in pharmacies, clinics, and hospital was 3.6, 3.1, and 3.2 weeks, respectively, and the overall mean duration was 3.4 weeks. From these data, we emphasize that most patients were not vaccinated and had no self-medication before. For example, at pharmacies, the number of patients being not vaccinated was 442 , whereas modestly lower than that, 318 people were treated without self-medication. These figures in clinics were significantly smaller: 115 and 79 patients, respectively.

\section{$\mathrm{COI}$ of ILI and diagnosed influenza}

Total COI of ILI in pharmacies was predicted to be US\$26,446.38 in March 2016 (US\$1 =21,892 Vietnam Dong [VND]) (Table 2). ${ }^{16}$ The mean cost per patient was US $\$ 58.00$ ranging from US\$2.41 to US\$558.84 and the medium cost per patient was US $\$ 41.82$. The total cost included nearly equal percentage of total direct cost and total indirect cost, of which $49.4 \%$ was total direct cost and $50.6 \%$ was total indirect cost. In reference to the components of different costs, the main component in direct cost was direct non-medical cost which took up a percentage of $39.5 \%$ in the total cost.

In clinics, the total cost of treatment for ILI patient was US $\$ 23,985.20$. The total cost consists of total direct and total indirect costs. A significant difference was found between the total direct and total indirect costs. The total direct cost was US $\$ 20,059.17$ and accounted for $83.6 \%$, whereas the total indirect cost was only US $\$ 3,926.03$ and accounted for $16.4 \%$. Of the total cost, nearly $12.6 \%$ of the cost was direct medical cost, whereas $71.1 \%$ was direct-non medical cost. Thus, the average cost of treatment for ILI patient was US\$158.84 and the median cost per case was US\$108.42. Differences were 
Table I Demographics of the surveyed subjects

\begin{tabular}{|c|c|c|c|c|c|c|c|}
\hline \multirow[t]{2}{*}{ Characteristic } & \multicolumn{2}{|c|}{ Pharmacy } & \multicolumn{2}{|c|}{ Clinics } & \multicolumn{2}{|c|}{ Hospital } & \multirow{2}{*}{$\begin{array}{l}\text { Total } \\
n=658\end{array}$} \\
\hline & $n=456$ & $\%$ & $n=15$ & $\%$ & $n=5$ & $\%$ & \\
\hline \multicolumn{8}{|l|}{ Age (years) } \\
\hline Mean \pm SD & \multicolumn{2}{|c|}{$33.8 \pm 17.4$} & \multicolumn{2}{|c|}{$6.1 \pm 4.3$} & \multicolumn{2}{|c|}{$12.4 \pm 13.6$} & $25.8 \pm 19.4$ \\
\hline $0-14$ & 62 & 13.5 & 139 & 92.1 & 38 & 74.5 & 239 \\
\hline $15-24$ & 95 & 20.7 & 12 & 7.9 & 6 & 11.8 & 113 \\
\hline $25-64$ & 283 & 61.8 & - & - & 6 & 11.8 & 289 \\
\hline$>64$ & 16 & 3.5 & - & - & I & 2.0 & 17 \\
\hline \multicolumn{8}{|l|}{ Gender } \\
\hline Male & 187 & 41.0 & 60 & 39.7 & 28 & 54.9 & 275 \\
\hline Female & 269 & 59.0 & 91 & 60.3 & 23 & 45.1 & 383 \\
\hline \multicolumn{8}{|l|}{ Area of residence } \\
\hline Urban & 172 & 37.7 & 51 & 33.8 & 35 & 68.6 & 258 \\
\hline Rural & 284 & 62.3 & 100 & 66.2 & 16 & 31.4 & 400 \\
\hline \multicolumn{8}{|l|}{ Education } \\
\hline Illiterate & 29 & 6.4 & 102 & 67.5 & 27 & 52.9 & 158 \\
\hline Primary school & 40 & 8.8 & 34 & 22.5 & 9 & 17.6 & 83 \\
\hline Secondary school & 63 & 13.8 & 4 & 2.6 & 6 & 11.8 & 73 \\
\hline High school & 126 & 27.6 & 7 & 4.6 & 4 & 7.8 & 137 \\
\hline College & 93 & 20.4 & 2 & 1.3 & I & 2.0 & 96 \\
\hline Bachelor degree and above & 105 & 23.0 & 2 & 1.3 & 4 & 7.8 & 111 \\
\hline \multicolumn{8}{|l|}{ Occupation } \\
\hline Student & 105 & 23.0 & 58 & 38.4 & 15 & 29.4 & 178 \\
\hline Agriculturist & 20 & 4.4 & - & - & - & - & 20 \\
\hline Labor/employee & 96 & 21.1 & I & 0.7 & I & 2.0 & 98 \\
\hline Public employee/state enterprise & 44 & 9.6 & 3 & 2.0 & 1 & 2.0 & 48 \\
\hline Private employee & 96 & 21.1 & I & 0.7 & - & - & 97 \\
\hline Housewife & 24 & 5.3 & - & - & 1 & 2.0 & 25 \\
\hline Unemployed & 3 & 0.7 & - & - & 1 & 2.0 & 4 \\
\hline Kindergarten student & 31 & 6.8 & 84 & 55.6 & 32 & 62.7 & 147 \\
\hline Other & 37 & 8.1 & 4 & 2.6 & - & - & 41 \\
\hline \multicolumn{8}{|l|}{ Income per month (US\$) } \\
\hline Mean \pm SD & \multicolumn{2}{|c|}{$225.8 \pm 199.8$} & \multicolumn{2}{|c|}{$168.6 \pm 94.9$} & \multicolumn{2}{|c|}{$167.8 \pm 124.4$} & $208.2 \pm 177.8$ \\
\hline$<250$ & 275 & 60.3 & 135 & 89.4 & 37 & 72.5 & 447 \\
\hline 250 to $<400$ & 125 & 27.4 & 8 & 5.3 & 8 & 15.7 & $|4|$ \\
\hline 400 to $<550$ & 43 & 9.4 & 6 & 4.0 & 5 & 9.8 & 54 \\
\hline 550 to $<700$ & I & 0.2 & I & 0.7 & 1 & 2.0 & 3 \\
\hline$\geq 700$ & 12 & 2.6 & I & 0.7 & - & - & 13 \\
\hline Satisfied with their quality of life and in & & & & & & & \\
\hline$<250$ & 231 & 50.7 & 130 & 86.1 & 34 & 66.7 & 395 \\
\hline 250 to $<400$ & 117 & 25.7 & 7 & 4.6 & 7 & 13.7 & 131 \\
\hline 400to- $<550$ & 39 & 8.6 & 6 & 4.0 & 5 & 9.8 & 50 \\
\hline 550 to $<700$ & I & 0.2 & I & 0.7 & I & 2.0 & 3 \\
\hline$\geq 700$ & 10 & 2.2 & 1 & 0.7 & - & - & II \\
\hline Self-medication & & & & & & & \\
\hline Yes & 138 & 30.3 & 72 & 47.7 & 29 & 56.9 & 239 \\
\hline No & 318 & 69.7 & 79 & 52.3 & 22 & 43.1 & 419 \\
\hline Vaccination & & & & & & & \\
\hline Yes & 14 & 3.1 & 36 & 23.8 & 7 & 13.7 & 57 \\
\hline No & 442 & 96.9 & 115 & 76.2 & 44 & 86.3 & 601 \\
\hline History of influenza & & & & & & & \\
\hline Yes & 285 & 62.5 & 69 & 45.7 & 21 & 41.2 & 375 \\
\hline No & 171 & 37.5 & 82 & 54.3 & 30 & 58.8 & 283 \\
\hline Had for weeks & 3.6 & & 3.1 & & 3.2 & & 3.4 \\
\hline Appearance of first symptoms (days) & 2.7 & & 3.7 & & 3.5 & & 3.0 \\
\hline Average number of family members & 4.2 & & 4.1 & & 4.1 & & 4.2 \\
\hline
\end{tabular}




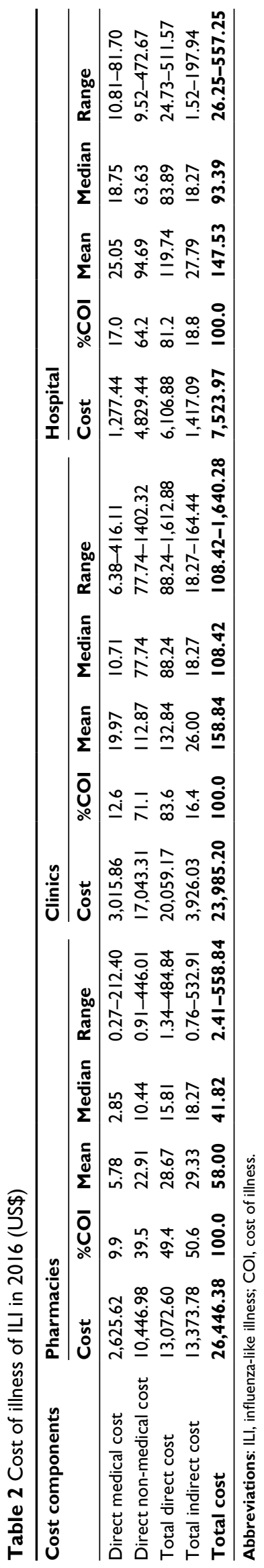

observed for costs among individual patients. The highest cost per case was US $\$ 1,640.28$, which was above 10-fold higher than the lowest cost per case (US\$108.42).

The total cost of treatment at a hospital was US\$7,523.97 which was combined by total direct cost (US\$6,106.88) which accounted for $(81.2 \%)$ and total indirect cost (US\$1,417.09) which contributed about $18.8 \%$. Of the total direct cost of US $\$ 6,106.88$, US $\$ 4,829.44$ was spent on direct non-medical cost and the remaining on direct medical cost. The results showed that the mean cost for each ILI patient was US $\$ 147.53$ and the median cost was US\$93.39. In contrast to the lowest cost per case (US\$26.25), it was noticed that the highest cost was much higher with US\$557.25 per ILI case. Comparison of direct costs among different components showed that the mean direct non-medical cost (US\$94.69) was higher than the mean direct medical cost (US\$25.05).

Of the three areas surveyed, the cost of ILI was found to be the lowest in hospital (US\$7,523.97) and highest in pharmacies (US\$26,446.38). Furthermore, the total direct cost was highest in the clinics (83.6\%) and total indirect cost in pharmacies (50.6\%) compared to other factors. The lowest mean cost per case was observed in pharmacies (US\$58.00). In contrast, each ILI patient in clinics spent $\sim$ US $\$ 158.84$. Comparison of the costs in pharmacies, clinics, and hospital showed that the median cost spent by those in clinics (US\$108.42) was significantly higher than that spent by others in hospital and clinics. Moreover, the range of total cost in clinics also varied from the lowest (US\$108.42) to the highest (US\$1,640.28).

\section{COI for different age groups}

As can be seen from Table 3, the mean age \pm SD of patients interviewed in the pharmacies, clinics, and the hospital was $33.82 \pm 17.44$ years, $6.10 \pm 4.35$ years, and $12.41 \pm 13.63$ years, respectively. Regarding the age of patients in each health facility, most patients at clinics and hospital were less than 14 years, accounting for $92.1 \%$ and $74.5 \%$, respectively. Meanwhile, most patients at pharmacies were adults (62.1\%). Table 3 also shows that there were no patients $>25$ years old in clinics, and there was only one senior patient older than 64 years in hospital.

In general, patients ( $0-14$ years) who underwent treatment at clinics had the highest COI with US\$160.36 per case, ranging from US $\$ 29.82$ to US $\$ 1,640.28$. However, patients aged between 15 and 24 years who underwent treatment in pharmacies had the lowest COI with US $\$ 45.33$ per patient, ranging from US $\$ 4.22$ to US\$310.51. In other respects, there was a high difference in the total costs that patients aged from 
25 to 64 years old had to pay: the lowest cost was US\$2.41 while the highest cost was US\$558.84 per patient.

Of the 456 ILI patients interviewed in pharmacies, 283 patients were 25-64 years old and 95 patients $15-24$ years old. In pharmacies, the COI differed for different age groups. The mean cost of treatment for patients below 14 years was the highest (US\$77.44), whereas the cost for other age groups ranged from US\$45.33 to US\$58.81. The median cost of treatment was also highest (US\$52.68) for patients below14 years, whereas it was lowest (US\$29.11) for patients who were 15-24 years old. Otherwise, patients aged from 25 to 64 years old had the widest range of treatment cost (US\$2.41 to US\$558.84).

Only one patient below 24 years chose a clinic to treat their illness. Furthermore, majority of the patients were children below 14 years (139 out of 151 patients), whose mean expense was US\$160.36. On the contrary, the expense of patients aged from 15 to 24 years was US\$141.22 (range: from US\$47.92 to US\$354.32). Children below 14 years had the highest median COI (US\$108.68) and the widest range of ILI cost (US\$29.82 to US\$1,640.28).

Most of the patients in hospital were children below 14 years (38 out of 51 total patients) and the cost of treatment was US $\$ 160.47$ per case. The mean cost of treatment for patients aged 15-24, 25-64, and $>64$ years was US $\$ 89.08$, US\$136.62, and US\$72.06, respectively. The median cost of treatment for ILI patients below 14 years, and 15-24 and 25-64 years was US\$114.75, US\$74.40, and US\$70.69, respectively. The cost of treatment varied more for children below 14 years, which ranged from US\$26.25 to US\$557.25.

\section{Discussion}

This study is a pioneer research in Vietnam that gives a comprehensive view of the features and costs of treatment of both ILI and clinically diagnosed influenza in four age groups: children ( $0-14$ years), youth (15-24 years), adults (25-64 years), and elderly ( $\geq 64$ years). The present survey found that in pharmacies adult patients were found to be mostly affected by ILI as there were 283 cases in March 2016. Meanwhile, in both clinics and hospital, children were mainly affected, with their population in the said places being 139 and 38, respectively. Besides, over half of the 658 patients who were interviewed indicated that they have had influenza at least once in their lifetime.

This study also found that the ratio of males and females with incidence of ILI was not even. In fact, the total number of women interviewed was generally lower than that of men: 269 females out of the total 456 interviewees in pharmacies, 91 out of 151 in clinics, and 23 out of 51 in hospital. In 
addition, the majority of subjects were female (58.2\%) with a mean age of 25.8 years applied for all feminine interviewees in pharmacies, clinics and hospital. In addition, out of the 658 patients, 57 were vaccinated for influenza. Similar to this study, Keech et al included only $55 \%$ of females in their study with a mean age of 34.2 years, and out of the 411 participants enrolled, only 11 had been reported to be vaccinated for influenza. ${ }^{17}$

Another fascinating discovery showed that urban residents were known to experience ILI more than their rural counterpart due to the dense population and degraded air quality in big cities. Consequently, contagiousness and transmission of influenza was likely to be more rapid in big cities compared to the rural areas. Hence, the morbidity was likely to be high in urban regions. ${ }^{18}$ Nevertheless, throughout this survey, the percentage of patients in rural areas appeared to be higher as they had ideal conditions to attend the survey.

Of the 658 patients, the number of students and kindergarten children accounted for 178 (27.1\%) and 147 $(22.3 \%)$, respectively, which was higher compared to other age groups. And most of the patients also were illiterate (158/658), high school students (137/658), and those with bachelor degree and above (11/658). In an occupation analysis carried out in South China in 2007, it was found that preschool children and students suffered the most from ILI with their incidence rate per annum of $42.08 \%$ and $10.73 \%$, respectively. Furthermore, it is believed that education level plays a role in the transmission of ILI, as the annual ILI incidence would likely decrease with an increase in education with a maximum value of 44.2 cases per 100 people in preschool children and a minimum value of 3.18 cases per 100 people in individuals having a bachelor degree or higher education. ${ }^{11}$

Average cost associated with ILI was US\$88.09 per case. But a study conducted in Hong Kong showed the average cost to be US\$36 per ILI episode. ${ }^{19}$ Moreover, this study shows that direct costs contributed in a different way to the total COI compared to indirect costs in different health care centers (e.g., pharmacies, private clinics, and hospital). At pharmacies, indirect cost was higher than the direct cost, whereas in clinics and hospital, a contrast result was found. This might be due to the fee that patients have to pay in clinics and hospital such as for a laboratory test or pharmaceuticals required to treat severe symptoms. Results obtained from all the three areas surveyed show that direct non-medical cost was dominant compared to direct medical cost, accounting for $39.5 \% \mathrm{COI}$ in pharmacies, $71.1 \%$ in clinics, and $64.2 \%$ in hospital.
Indirect cost was calculated by the cost of day loss caused by illness. According to this study, indirect cost was US\$27.49 per episode, which was calculated considering the total number of patients. But taking into account each individual study area, the average cost was calculated to be equal to US\$29.33 per case in pharmacies, US\$26.00 in clinics, and US\$27.79 in hospital. Based on the results of this study, it has been concluded that the value of direct medical cost was US\$10.51, whereas a study in South China showed that the direct medical cost of ILI patient was US\$22.69. ${ }^{11}$

Regarding age groups, the average total cost for children under 14 years old was much higher than that for the other age groups. The mean cost of treatment for children under 14 years old was US\$77.44 in pharmacies, US\$160.36 in clinics, and US\$160.47 in hospital. Moreover, a twofold increase in the mean and median values of COI was observed for clinics and hospital compared to pharmacies. Besides, the average total cost per episode was US\$138.87 for children and US\$59.11 for adults. As a matter of fact, it is important that measures to control and prevent influenza cases should be adopted in Vietnam with a special focus on children. By contrast, Ehlken et al's study in Germany showed that the total average mean cost per episode was estimated to be $€ 105$ for children and $€ 514$ for adults. ${ }^{9}$

The limitations of this study were, firstly, the short survey period and inappropriate time of the study (i.e., the emergence of influenza pandemic was rare at the time of the study). Therefore, the result might not accurately reflect the situation of influenza in Vietnam. Secondly, the survey was only conducted in Southern Vietnam. Thus, the results are not to be used to evaluate the overall situation of Vietnam.

\section{Conclusion and limitations}

This study has attempted to show the economic impacts that ILI and clinically diagnosed influenza are likely to have on society. The results clearly show that the COI was the reason for the economic burden of influenza patients and their families. Furthermore, this study provides a database for future research, programs, and policies that can be adopted for influenza or ILI in Vietnam. This study clearly emphasizes that the burden of influenza could be deterred if the efficiency or the coverage of influenza vaccination is enhanced.

This study has some limitations. Some health facilities and patients refused to participate in this research due to their privacy. Even though the database is large, it would not represent the entire Vietnamese population. In addition, the database would be more precise if the duration of the study covered the flu season. 


\section{Acknowledgment}

We would like to address our most sincere gratitude to the Faculty of Pharmacy, Mahidol University, for providing us with research facilities, as well as pharmacies, clinics, and the hospital in Southern Vietnam for permitting us to conduct our study at their sites.

\section{Disclosure}

The authors report no conflicts of interest in this work.

\section{References}

1. Nicholson K. Clinical features of influenza. Semin Respir Infect. 1992;7(1):26-37.

2. World Health Organization. Influenza (Seasonal) Fact Sheet No. 211, March 2014. Geneva: Word Health Organization; 2014.

3. Nordqvist C. Flu: symptoms, Treatment, and prevention. Medicalnewstoday (MNT). 2016. Available from: http://www.medicalnewstoday. com/articles/15107.php. Accessed May 22, 2016.

4. Fleming DM, Zambon M, Bartelds AI, de Jong JC. The duration and magnitude of influenza epidemics: a study of surveillance data from sentinel general practices in England, Wales and the Netherlands. Eur J Epidemiol. 1999;15(5):467-473.

5. Molinari NA, Ortega-Sanchez IR, Messonnier ML, Thompson WW, Wortley PM, Weintraub E, Bridges CB. The annual impact of seasonal influenza in the US: measuring disease burden and costs. Vaccine. 2007;25(27):5086-5096.

6. Newall AT, Scuffham PA. Influenza-related disease: the cost to the Australian healthcare system. Vaccine. 2008;26(52):6818-6823.

7. Ortega-Sanchez IR, Molinari NA, Fairbrother G, et al. Indirect, outof-pocket and medical costs from influenza-related illness in young children. Vaccine. 2012;30(28):4175-4181.
8. Bilcke J, Coenen S, Beutels P. Influenza-like-illness and clinically diagnosed flu: disease burden, costs and quality of life for patients seeking ambulatory care or no professional care at all. PLoS One. 2014;9(7):e102634.

9. Ehlken B, Anastassopoulou A, Hain J, Schröder C, Wahle K. Cost for physician-diagnosed influenza and influenza-like illnesses on primary care level in Germany - results of a database analysis from May 2010 to April 2012. BMC Public Health. 2015;15:578.

10. Yin JK, Salkeld G, Lambert SB, et al. Estimates and determinants of economic impacts from influenzalike illnesses caused by respiratory viruses in Australian children attending childcare: a cohort study. Influenza Other Respir Viruses. 2013;7(6):1103-1112.

11. Guo R, Zheng H, Li J, Sun LM, Li LH, Lin JY, He JF. A populationbased study on incidence and economic burden of influenza-like illness in south China, 2007. Public Health. 2011;125(6):389-395.

12. Nam GSOoV. Report of monthly mean air temperature in 2015. Available from: http://www.gso.gov.vn/Default.aspx?tabid=217. Accessed May 29, 2016

13. Nam GSOoV. Report of area, population and population density 2014. Available from: http://www.gso.gov.vn/Default.aspx?tabid=706\& ItemID=13412. Accessed May 29, 2016.

14. Wayne W. Daniel, Cross CL. Biostatistics: Basic Concepts and Methodology for the Health Sciences. Hoboken: John Wiley; 2010.

15. World Health Organization. A Manual for Estimating Disease Burden Associated with Seasonal Influenza. Geneva: World Health Organization; 2015.

16. Vietnam MoFo. Exchange Rate USD Dollar/VND Dong. Available from: http://www.mof.gov.vn/. Accessed Jan 20, 2016.

17. Keech M, Scott A, Ryan P. The impact of influenza and influenza-like illness on productivity and healthcare resource utilization in a working population. Occup Med. 1998;48(2):85-90.

18. Lofgren E, Fefferman NH, Naumov YN, Gorski J, Naumova EN. Influenza seasonality: underlying causes and modeling theories. JVirol. 2007;81(11):5429-5436.

19. Fitzner KA, Shortridge K, McGhee S, Hedley A. Cost-effectiveness study on influenza prevention in Hong Kong. Health Policy. 2001;56(3):215-234.
ClinicoEconomics and Outcomes Research

\section{Publish your work in this journal}

ClinicoEconomics and Outcomes Research is an international, peerreviewed open-access journal focusing on health technology assessment, pharmacoeconomics and outcomes research in the areas of diagnosis, medical devices, and clinical, surgical and pharmacological intervention. The economic impact of health policy and health systems
Submit your manuscript here: https://www.dovepress.com/clinicoeconomics-and-outcomes-research-ioun

organization also constitute important areas of coverage. The manuscript management system is completely online and includes a very quick and fair peer-review system, which is all easy to use. Visit http://www.dovepress.com/testimonials.php to read real quotes from published authors. 